\title{
Brain-electric activity during eyes open Brahma Kumaris Rajayoga meditation.
}

\author{
Kanishka Sharma ${ }^{\mathrm{a}}$, Peter Achermann ${ }^{\mathrm{b}}$, Bhawna Panwar ${ }^{\mathrm{c}}$, Shrikant Sahoo ${ }^{\mathrm{c}}$, Ramakrishnan Angarai \\ Ganesan $^{\mathrm{a}}$, Roberto D. Pascual-Marqui ${ }^{\mathrm{b}}$, Pascal L. Faber ${ }^{\mathrm{b}}{ }^{*}$ \\ ${ }^{a}$ Medical Intelligence and Language Engineering (MILE) Lab, Electrical Engineering Department, Indian Institute of \\ Science, Bengaluru, India
}

${ }^{\mathrm{b}}$ The KEY Institute for Brain-Mind Research, Department of Psychiatry, Psychotherapy and Psychosomatics, University Hospital of Psychiatry, Zurich, Switzerland

${ }^{c}$ Spiritual Applications Research Centre (SpARC Wing) of Rajyoga Education and Research Foundation (RERF)

BrahmaKumaris, Mount Abu, India

${ }^{*}$ Corresponding author at:

The KEY Institute for Brain-Mind Research, University Hospital of Psychiatry, Lenggstr. 31, CH-8032 Zurich, Switzerland. E-mail address: pfaber@key.uzh.ch (P.L. Faber) / scholar.google.com/citations?user=pascalfaber

\section{Acknowledgements}

The authors would like to acknowledge the team members who supported in experimental recording.

\section{Abstract}

Brahma Kumaris Rajayoga's open eyed 'seed stage meditation' was studied in 52 meditators. Meditation stages: concentration on peace, realization of being a soul and communion with the Supreme Soul. Frequency domain brain electric source localization was used on multichannel EEG recordings to establish activation differences between meditation and open eyed, task-free resting. Additional exploratory analyses probed for effects of passing time from initial rest through meditation to final rest. Meditation showed reduced activity in delta and increased activity in low alpha frequencies. Affected brain areas comprised the following networks: central executive network, mirroring network, task-positive and task-negative network. These altered activations reflect the main cognitive-affective and behavioral specifics of seed stage meditation: attention modulation, self-related processing, experiencing the soul as a point of light between the eyes, endowing the soul with the properties (peacefulness) of the Supreme Soul. Future studies need to differentiate between the stages of the meditation.

\section{Keywords}




\section{Introduction}

Brahma Kumaris (BK) Rajayoga is a modern revival of the Indian Rajayoga (RY) system. Unlike most other RY systems, BK Rajayoga is not based on the Patanjalayoga system and has little to do with it (Birch, 2013). Rajayoga as taught by the Prajapita Brahmakumaris World Spiritual University and is popularized as a way for self-realization and the realization of the supreme almighty. It does not rely on rituals or mantras and can be practiced by anyone anywhere at any time. The most common meditation practice within this tradition is 'seed stage meditation'. Practitioners believe that through this practice they seek the intellectual and loving communion of the soul with the Supreme Soul (Brahmakumaris, 1986). This meditation follows several steps and moves through different stages (Ramesh, Sathian, Sinu, \& Kiranmai, 2013; Telles \& Desiraju, 1993). Sitting with eyes open, in a comfortable posture, for example in an armchair, the practitioner gazes at a meaningful symbol, such as a picture depicting the Supreme Soul as a radiating point of light or just imagines the emergence of soul in between the two eye brows facing a neutral wall. The meditation itself goes through the following stages using appropriate autosuggestions to keep the mind focused on task during the process, thus avoiding the mind from wandering. Practice begins with sitting quietly and relaxed, followed by the stage of concentration, when the practitioner uses auto-suggestions to settle into a feeling of peace. This meditation can also be done using feelings of purity, love, joy, power or wisdom. In the present study, only the feeling of peace was used. The practitioner may either try to produce the feeling of peacefulness in the moment or bring forth this feeling through recalling it from an autobiographical memory. This feeling of peace is the foundation for the next stage, namely soul consciousness. The practitioner reminds him-/herself that he/she is a soul, a sparkling light between his/her eyes. The last stage is described by the practitioners as the connection (a conversation) of the soul with the Supreme Soul, a bodyless light source with perfect qualities of peace. The practitioner imagines receiving these qualities from the Supreme Soul and letting them permeate the soul. When successful, this culminates in absorption. The practitioner's mind is totally calm, and there is little to no active guiding of the intellect; only calmness and absorption. Soul consciousness is a progression away from everyday concerns, away from the body, towards the realization of being a soul and the connection of this soul with the Supreme Soul and ultimately the absorption within it.

On a side note, seed stage meditation is practiced with open eyes for two reasons (Ramsay, Manderson, \& Smith, 2010): First, the practitioners thus exercise their faculty to meditate anywhere irrespective of what is going on around them. Second, it is believed to enable direct communication between souls through 'drishti' ('vision') such as exchanging positive feelings.

Several attempts have been put forth in the literature to categorize meditation practices. The most common classification systems distinguish between focused attention (FA), open monitoring (OM) and automatic self-transcendence practices (Lutz, Slagter, Dunne, \& Davidson, 2008; Raffone \& Srinivasan, 2010; Travis \& Shear, 2010). Josipovic (2010) proposed to include nondual awareness as a defining characteristic of some practices. Nash and Newberg (2013) categorize practices as belonging to a cognitive, affective and null domain. The respective practices foster an enhanced cognitive, affective, or non-cognitive/non-affective state. In order to allow for a better understanding of how the different practices might foster 
Sharma, Achermann, Panwar, Sahoo, Angarai Ganesan, Pascual-Marqui, Faber: Brain-electric activity during eyes open

well-being, Dahl et al. (2015) proposed a classification distinguishing between attentional, constructive, and deconstructive families of practices, i.e. practices that cultivate meta-awareness, enhance cognitive and affective patterns which increase well-being or focus on self-inquiry respectively.

How does BK Rajayoga meditation (BKRYM) fit into these schemes? Rajayoga meditation has strong elements of an FA meditation as it needs concentration to guide the mind through the different stages, focusing on the soul (i.e. a point of light between the eyebrows) and the qualities of the Supreme Soul. This process of observing soul as a different entity from body but residing in itself can be categorized as self-monitoring. Following the classification of Nash \& Newberg (2013), this would be the cognitive domain. As it also has a strong affective component with the practitioner seeking the feeling of peacefulness, the affective domain is also strongly present. Within the classification of Dahl et al. (2015) , BKRYM most strongly fits into the constructive family of practices, as it targets a change in perspective, a cognitive reappraisal of oneself as a soul that is pure in its qualities as are the souls of all human beings (see also Nair, Sasidharan, John, Mehrotra, \& Kutty, 2017).

In general, regular meditation practice has many potential benefits (for a review, see Keng, Smoski, \& Robins, 2011), such as increased subjective well-being, reduced psychological symptoms and emotional reactivity, and improved behavioral regulation. For a review on the benefits of yoga meditation in particular (including RY), see Rajoria and Singh (2017). Among the reported benefits of the practice of BK Rajayoga specifically are the following: improved basic cardiorespiratory functions (Sukhsohale \& Phatak, 2012); reduced anxiety and depression scores (Kiran, Chalana, Arora, \& Girgila, 2014); higher self-satisfaction and happiness in life compared to a non-meditator group (Ramesh et al., 2013); less neurotic symptoms and higher scores on hope and happiness (Misra, Gupta, Alreja, \& Prakash, 2013); improved general well-being as measured with a quality of life questionnaire (WHO-QOL) after one year of practice (Meshram \& Meshram, 2016) and significant increase of IQ in a group of 42 ADHD children after 3 months of practice (Naik, Patel, Biswas, \& Verma, 2016). BK Rajayoga as other spiritual paths is thought to help generate resilience through the cultivation of meaning and self-transformation based on the respective spiritual guidelines (Ramsay \& Manderson, 2011).

Not much is known about the brain electric mechanisms sub-serving the different states of the meditation practice of BK Rajayoga. A popular one-minute meditation in contrast with resting was explored in long-term BK Rajayoga practitioners, short term practitioners and meditation naïve subjects. This study reported increased theta and alpha band-power in the EEG for long-term and short-term practitioners respectively during meditation compared to resting (Nair et al., 2017), also longterm meditators reliably shifted between resting and meditation states, short-term meditators less reliably and controls were unable to do so. Another study exploring a 10-minute practice of meditation found changes in theta and lower alpha band and higher alpha-asymmetry in meditators during meditation compared to controls during resting (Sharma, Chandra, \& Dubey, 2018). The activity of the default mode network (DMN) during BKRYM and resting compared to resting in control subjects was studied in a study using simultaneous EEG-fMRI recordings (Panda et al., 2016). Increased duration and occurrence of the EEG microstates corresponding to DMN activation was reported as well as an increase in EEG spectral power in the alpha, theta and beta bands (Panda et al., 2016). 
Sharma, Achermann, Panwar, Sahoo, Angarai Ganesan, Pascual-Marqui, Faber: Brain-electric activity during eyes open

In order to prepare the ground for future investigations about how physiological and psychological health benefits might be associated with the brain electric specifics of the BKRYM practice, we investigated the brain electrical underpinnings of this meditation practice compared to task free resting within a large group of experienced practitioners. We used frequency domain source localization to detect intracortical electrical activity changes occurring during meditation. Based on the description above of the cognitive and affective particulars of this practice, we expected brain areas involved in attention modulation as well as emotion and memory processing to show alterations in electrical activity. Consequently, we expected the central executive network (CEN) and the task-positive network to show increased activation and the default mode network to show decreased activation.

\section{Materials \& methods}

\subsection{Participants}

Fifty-two meditators (age: $42.0 \pm 10.1$ years, range: $25-59$; meditation experience: $17.5 \pm 10.8$ years, range: $4-43 ; 14$ females) were recruited and recorded during winter season when followers from across the world visit the headquarters of Brahma Kumaris.

\subsection{EEG recordings}

The EEG recordings took place at the International Centre for Higher Learning, Academy for a Better World, Brahma Kumaris, Gyan Sarovar, Mount Abu, India (1722 m above sea level). The recordings were performed in a small normally lit room with the participants sitting upright and either cross-legged on a couch or on the couch border with their feet on the ground. They were facing a neutral, coffee-colored tapestry on the wall. The experimenter controlled the recording from a small adjacent room containing the recording computer and allowing easy view of the participant through a clear glass window. Based on the 10-10 electrode placement system (Nuwer et al., 1998), 61 EEG channels were recorded at the following locations: Fp1, Fpz, Fp2, F7, F3, Fz, F4, F8, FC5, FC1, FC2, FC6, T7, C3, Cz, C4, T8, CP5, CP1, CP2, CP6, P7, P3, Pz, P4, P8, POz, O1, O2, AF7, AF3, AF4, AF8, F5, F1, F2, F6, FC3, FCz, FC4, C5, C1, C2, C6, CP3, CP4, P5, P1, P2, P6, PO5, PO3, PO4, PO6, FT7, FT8, TP7, TP8, PO7, PO8, Oz. All the channels were referenced to CPz, and AFz was used as ground. Horizontal left EOG was also recorded. The recordings were performed using a 64 channel ANT neuro mylab@ system. EEG was recorded at $500 \mathrm{~Hz}$. Impedance for all EEG and EOG channel was lower than $10 \mathrm{~K}$ Ohms to ensure data quality. The EEG amplifier along with the acquisition system (laptop) were running on battery and were not connected to the mains during data recording. Band pass filter was applied from 0.3 to $75 \mathrm{~Hz}$ in the recording software as was a $50 \mathrm{~Hz}$ notch filter. 


\subsection{Recording conditions}

The recordings adhered to the following protocol:

- Initial rest: 3 min with eyes open (instruction: 'sit quietly without meditating')

- Initial rest: 3 min with eyes closed (instruction: 'close your eyes and sit quietly without meditating')

- $\quad 30$ min of Rajayoga seed stage meditation with eyes open (shifting the awareness from the visible world to "the soul and its peaceful nature' and then connecting to the 'Supreme Soul')

- Final rest: 3 min with eyes open (instruction: 'sit quietly without meditating')

- Final rest: 3 min with eyes closed resting (instruction: 'close your eyes and sit quietly without meditating')

During the 30 min of meditation the participants were prompted 4 to 5 times at random intervals via an acoustic tone to mentally note what was going through their minds. The whole protocol was timed using digital stopwatch to ensure proper length of data segments.

For the present report, mainly initial rest with eyes open was compared to meditation. Additional analyses were done to test for effects of passing time: the meditation data was cut into thirds to test for changes over time during meditation and meditation as well as initial rest (eyes open) were also compared to final rest (eyes open).

\subsection{Data conditioning}

The preprocessing of the EEG data first consisted of down-sampling the data to $128 \mathrm{~Hz}$ using spline interpolation. Then, 1 min of EEG was removed after each tonal prompt to avoid the inclusion of non-meditation epochs. First, eye movement artifacts were corrected using independent component analysis where necessary and then, the remaining eye, muscle, sweat and technical artifacts were marked through visual inspection. The EEG was segmented into 2-s epochs and all artifact-free epochs were exported for further analysis. The data was preprocessed using BrainVision Analyzer version 2.1.2 (www.brainproducts.com). The meditation session was cut into thirds to be able to check for time effects. To account for the difference in the recording duration between meditation and rest, a random selection of 50 artifact free epochs were collected for initial and final rest as well as for each third of the meditation (resulting in a total of 150 epochs for the complete meditation session).

\subsection{Data analysis}

The 50 preprocessed, artifact-free 2-s epochs per condition (150 for combined meditation thirds) were subjected to source localization analyses using exact low resolution brain electromagnetic tomography (eLORETA, Pascual-Marqui, 2007), freely available at http://www.uzh.ch/keyinst/loreta.htm. eLORETA is a genuine inverse solution that shows exact, zero error localization even in the presence of measurement and structured biological noise (Pascual-Marqui, 2007). The analysis procedure follows the LORETA functional tomography analysis approach (Pascual-Marqui et al., 1999; Pascual-Marqui, Michel, \& Lehmann, 1994). Applying the procedure delineated in Frei et al. (2001), cross-spectra (128 discrete frequencies, 
Sharma, Achermann, Panwar, Sahoo, Angarai Ganesan, Pascual-Marqui, Faber: Brain-electric activity during eyes open

from $0.5-64.0 \mathrm{~Hz}$, at $0.5 \mathrm{~Hz}$ frequency resolution) were computed for all available epochs and averaged per condition and participant and then transformed into eLORETA images (with 6239 cortical voxels at a spatial resolution of $5 \mathrm{~mm}^{3}$ ). For all comparisons between and within conditions, paired t-statistics were computed on the log-transformed current density values at each voxel. Corrections for multiple testing (over all 6239 voxels and 128 frequencies) using nonparametric randomization (Nichols \& Holmes, 2002) were applied. Significant LORETA voxels were attributed to their corresponding Brodmann areas (BAs) based on their coordinates in MNI space (Evans \& Collins, 1993).

\section{Results}

\subsection{Meditation vs initial rest}

Frequencies showing significant differences between meditation and initial rest in at least 15 voxels were found in two frequency ranges: the delta range between 0.5 and $4.0 \mathrm{~Hz}$ and the range between 7.0 and $9.5 \mathrm{~Hz}$, which we consider as 'low alpha' (Klimesch, Doppelmayr, Schwaiger, Auinger, \& Winkler, 1999). The cluster of significant voxels was largest at 1.5 $\mathrm{Hz}$ for delta and at $9.0 \mathrm{~Hz}$ for low alpha activity.

Meditation showed decreased delta activity compared to initial rest in a large cluster encompassing bilateral prefrontal (BAs 9, 10, 46), orbitofrontal (left BAs 11, 47) and dorsolateral areas (BA 46), the anterior insula (BA 13), the frontal eye fields (BA 8), Broca's area (BAs 45, 44) and extending to the premotor cortex (BA 6) and in the primary motor cortex (BA 4), the somatosensory cortex (BAs 1, 2, 3 and adjacent BA 43), the precuneus (BA 7) and parietal areas (left BAs 5, 40) as well as temporal areas (BAs 20, 21, 22, 41, 42).

Meditation revealed increased activity compared to initial rest in low alpha frequencies. These increases were found bilaterally in the cingulate cortex (BAs 23, 24, 29, 30, 31), the parahippocampal and fusiform gyrus (BAs 27, 28, 36, 37), the superior and inferior temporal gyrus (BAs 22, 20), Wernicke's area (BAs 39, 40) and the auditory cortex (BAs 41, 42 right), the insula (BA 13), the associative and secondary visual cortices (BA 18, 19) and precuneus (BA 7).

Figure 1 illustrates these findings with cortical slices through difference maxima between conditions per frequency. The number of voxels significantly differing between conditions in the delta and low alpha range are listed in Table 1 (left) per Brodmann area and in Table 2 per anatomical structure.

No significant differences between meditation and initial rest were present in other frequency ranges, i.e. the theta, upper alpha, beta, and gamma frequencies. 
Sharma, Achermann, Panwar, Sahoo, Angarai Ganesan, Pascual-Marqui, Faber: Brain-electric activity during eyes open

A.

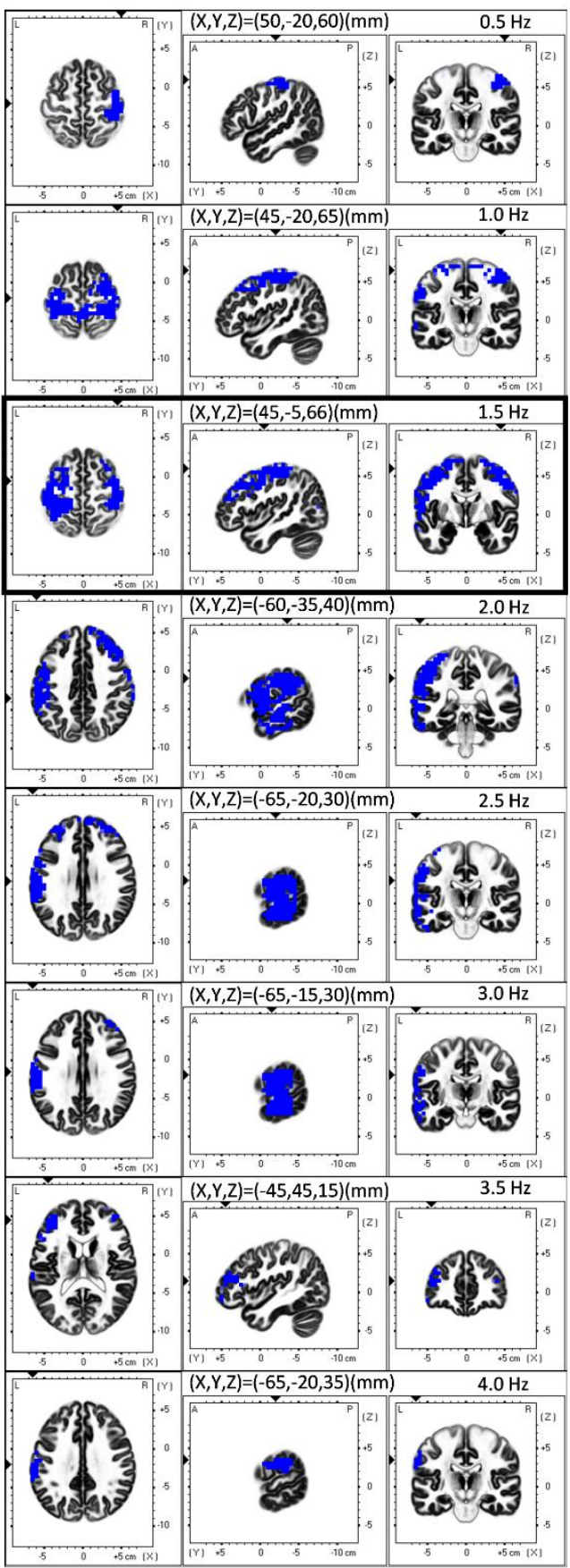

B.

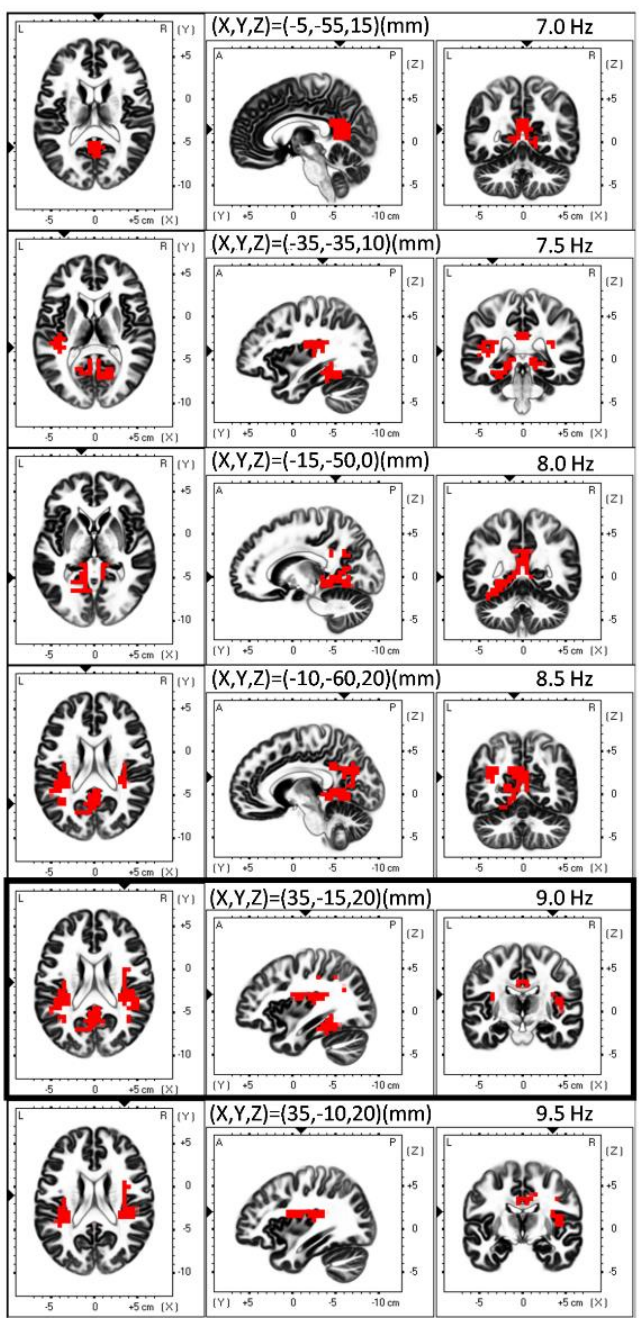

Fig. 1. Frequencies showing significant differences between meditation and initial rest. Decreases (blue) during meditation were seen in delta frequencies (A.) Increases (red) during meditation were seen in low alpha frequencies (B.). Shown are slices (transverse, sagittal and coronal from left to right) through the voxel of maximal difference (indicated by black triangles) between meditation and initial rest per frequency. The MNI coordinates of the voxel of maximal difference are indicated on top of each sagittal slice, and the frequency on top of the coronal slice. Slices with bold borders indicate the frequency in each frequency range with the highest number of significant voxels. 
Sharma, Achermann, Panwar, Sahoo, Angarai Ganesan, Pascual-Marqui, Faber: Brain-electric activity during eyes open

Table 1. Number of voxels per Brodmann area significantly differing between meditation and initial and final rest for specific frequency / frequency ranges showing at least 15 significant voxels in each associated frequency.
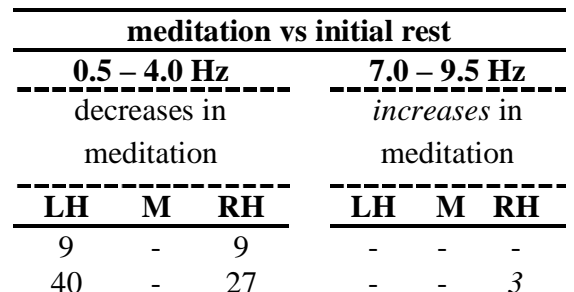

\begin{tabular}{c}
\hline BA \\
\hline $\mathbf{1}$ \\
2 \\
3 \\
4 \\
5 \\
6 \\
7 \\
8 \\
9 \\
10 \\
11 \\
13 \\
17 \\
18 \\
19 \\
20 \\
21 \\
22 \\
23 \\
24 \\
27 \\
28 \\
29 \\
30 \\
31 \\
32 \\
35 \\
36 \\
37 \\
38 \\
39 \\
40 \\
41 \\
42 \\
43 \\
44 \\
45 \\
46 \\
47 \\
\end{tabular}

$34 \quad-93$

$30 \quad-\quad 46$

4

$$
11-2
$$$$
-\quad-\quad-
$$$$
31
$$$$
63
$$$$
60 \quad-\quad 10
$$$$
3-8
$$$$
\begin{array}{lll}
12 & 1 & 19
\end{array}
$$$$
4 \quad 5 \quad 15
$$$$
7-9
$$$$
\begin{array}{lll}
3 & - & 1 \\
5 & - & 4
\end{array}
$$$$
30 \quad 3 \quad 35
$$$$
\begin{array}{lll}
56 & 1 & 53
\end{array}
$$$$
\begin{array}{ccc}
5 & - & 1 \\
10 & - & 10
\end{array}
$$$$
28-15
$$$$
18-3
$$$$
3-8
$$$$
17 \quad-\quad 21
$$

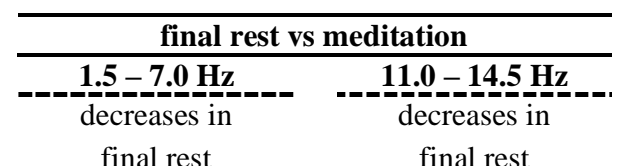

\begin{tabular}{ccc}
\hline $\mathbf{L H}$ & $\mathbf{M}$ & $\mathbf{R H}$ \\
\hline 7 & - & 9 \\
42 & - & 46
\end{tabular}

\begin{tabular}{|c|c|c|}
\hline $\mathbf{L H}$ & $\mathbf{M}$ & $\mathbf{R H}$ \\
\hline 4 & & 2 \\
\hline
\end{tabular}

$67 \quad-\quad 56$

59

40

$\begin{array}{lll}185 & 12 & 122\end{array}$

$\begin{array}{lll}186 & 25 & 189\end{array}$

$\begin{array}{lll}23 & 8 & 36\end{array}$

$38 \quad 3 \quad 9$

$\begin{array}{ccc}49 & - & 31 \\ 40 & 1 & 42 \\ 40 & 9 & 41 \\ 163 & 13 & 150 \\ 98 & 10 & 85 \\ 10 & 7 & 9 \\ - & - & 2\end{array}$

\begin{tabular}{cc}
\hline \multicolumn{2}{c}{ final rest vs initial rest } \\
\hline 1.5-7.0 Hz & $\mathbf{2 6} \mathrm{Hz}$ \\
\hline decreases in & -0 dereases in
\end{tabular}

final rest

final rest

\begin{tabular}{|c|c|c|c|c|c|}
\hline LH & $\overline{\mathbf{M}}$ & $\mathbf{R H}$ & $\mathbf{L H}$ & $\mathbf{M}$ & $\mathbf{R H}$ \\
\hline 9 & - & 8 & 1 & - & - \\
\hline 43 & - & 42 & 6 & - & - \\
\hline 72 & - & 54 & 17 & - & - \\
\hline 69 & 1 & 70 & 8 & - & - \\
\hline 40 & 9 & 41 & 14 & - & 9 \\
\hline 261 & 13 & 275 & 15 & - & - \\
\hline 186 & 25 & 198 & 15 & - & 78 \\
\hline
\end{tabular}$$
12
$$$$
36
$$

$\begin{array}{lll}145 & 28 & 80\end{array}$$$
\begin{array}{lll}
180 & 2 & 78
\end{array}
$$$$
\begin{array}{ccc}
- & - & - \\
- & - & - \\
6 & - & 4 \\
15 & 15 & 22 \\
39 & 13 & 46
\end{array}
$$$$
\begin{array}{lll}
- & - & - \\
- & - & -
\end{array}
$$$$
\begin{array}{lll}
74 & 9 & 91
\end{array}
$$$$
124 \quad 9 \quad 134
$$$$
56 \quad-\quad 51
$$$$
\begin{array}{ccc}
- & - & 6 \\
37 & - & 36
\end{array}
$$$$
\begin{array}{ll}
36 & 2 \\
141 & 28
\end{array}
$$$$
\begin{array}{lll}
141 & 28 & 142
\end{array}
$$$$
\begin{array}{lll}
179 & 2 & 170
\end{array}
$$$$
11 \text { - } 74
$$$$
12 \quad-\quad 101
$$$$
\begin{array}{lll}
12 & - & 85
\end{array}
$$$$
\begin{array}{lll}
10 & 8 & 11
\end{array}
$$$$
\begin{array}{lll}
44 & 13 & 58
\end{array}
$$$$
\begin{array}{lll}
3 & - & 3
\end{array}
$$$$
\begin{array}{ccc}
- & - & - \\
- & - & - \\
5 & - & 4 \\
32 & 3 & 19 \\
80 & 29 & 59 \\
17 & 4 & 26
\end{array}
$$$$
\begin{array}{ccc}
- & - & - \\
- & - & - \\
- & - & - \\
- & - & - \\
42 & 19 & 37 \\
22 & 4 & 28
\end{array}
$$$$
\begin{array}{lll}
43 & 9 & 37
\end{array}
$$$$
\begin{array}{ccc}
- & - & - \\
- & - & - \\
- & - & - \\
13 & - & 13 \\
49 & 10 & 35 \\
39 & 5 & 39
\end{array}
$$$$
23
$$$$
\begin{gathered}
- \\
70 \\
174
\end{gathered}
$$$$
\begin{gathered}
- \\
- \\
- \\
- \\
29 \\
147 \\
- \\
2 \\
- \\
- \\
- \\
6
\end{gathered}
$$$$
\begin{array}{ccc}
- & - & - \\
- & - & - \\
- & - & - \\
- & - & - \\
- & - & - \\
59 & - & 43
\end{array}
$$$$
\begin{array}{ccc}
- & - & - \\
1 & - & 15 \\
83 & - & 88 \\
- & - & 22 \\
77 & - & 60 \\
189 & - & 180 \\
13 & - & 27 \\
11 & - & 20 \\
12 & - & 11 \\
22 & - & 15 \\
14 & - & 27 \\
18 & - & 26 \\
1 & - & 28
\end{array}
$$

LH: left hemisphere, RH: right hemisphere, M: midline voxels. Numbers in italics indicate activity increases in the respective frequency / frequency range and condition, normal font numbers indicate activity decreases. 
Sharma, Achermann, Panwar, Sahoo, Angarai Ganesan, Pascual-Marqui, Faber: Brain-electric activity during eyes open

Table 2: Number of voxels per anatomical structure significantly differing between meditation and initial rest in 2 frequency ranges $(0.5-4.0 \mathrm{~Hz}$; decreases in meditation and 7.0-9.5 Hz; increases in meditation).

\begin{tabular}{|c|c|c|c|c|c|c|}
\hline anatomical structure & LH & $\mathbf{M}$ & RH & LH & $\mathbf{M}$ & RH \\
\hline Inferior Frontal Gyrus & 76 & - & 40 & - & - & - \\
\hline Medial Frontal Gyrus & 9 & - & 20 & - & - & - \\
\hline Middle Frontal Gyrus & 108 & - & 164 & - & - & - \\
\hline Superior Frontal Gyrus & 50 & - & 107 & - & - & - \\
\hline Precentral Gyrus & 149 & - & 115 & - & - & 2 \\
\hline Postcentral Gyrus & 160 & - & 103 & - & - & 5 \\
\hline Paracentral Lobule & 6 & 3 & 8 & - & - & - \\
\hline Anterior Cingulate & - & - & - & 6 & 6 & 22 \\
\hline Posterior Cingulate & - & - & - & 241 & 116 & 236 \\
\hline Parahippocampal Gyrus & - & - & - & 204 & - & 93 \\
\hline Transverse Temporal Gyrus & 12 & - & - & 20 & - & 10 \\
\hline Inferior Temporal Gyrus & 27 & - & - & 1 & - & - \\
\hline Middle Temporal Gyrus & 64 & - & 6 & 8 & - & 2 \\
\hline Superior Temporal Gyrus & 77 & - & 18 & 58 & - & 40 \\
\hline Insula & 8 & - & 1 & 125 & - & 129 \\
\hline Fusiform Gyrus & 9 & - & - & 80 & - & 22 \\
\hline Lingual Gyrus & - & - & - & 70 & - & 37 \\
\hline Precuneus & 1 & - & - & 92 & 30 & 38 \\
\hline Cuneus & - & - & - & 19 & - & 14 \\
\hline Inferior Parietal Lobule & 146 & - & 86 & 2 & - & 8 \\
\hline Superior Parietal Lobule & 10 & - & - & 7 & - & - \\
\hline
\end{tabular}

LH: left hemisphere, RH: right hemisphere, M: midline voxels. Numbers in italics indicate activity increases in the respective frequency range and condition, normal font numbers indicate activity decreases. 


\subsection{Temporal evolution during meditation}

Successive thirds of meditation did not differ, i.e. the beginning from the middle part and the middle from the final part. However, from the beginning to the final part of the meditation a decrease in low frequencies $(1.5-4.5 \mathrm{~Hz})$ in the motor (BAs 4, 6) and somatosensory (BAs 1, 2, 3, 5) cortices as well as parietal areas (BA 40) were observed (table 3). Some decreases occurred also at $26 \mathrm{~Hz}$, mainly in the left cingulate cortex (BAs 23, 24, 31; Table 3).

Table 3. Number of voxels per Brodmann area significantly differing between first and last third of meditation for frequencies / frequency ranges showing at least 15 significant voxels in each associated frequency.

\begin{tabular}{|c|c|c|c|c|c|}
\hline \multicolumn{6}{|c|}{ meditation third part vs first part } \\
\hline \multicolumn{3}{|c|}{$\begin{array}{c}\mathbf{1 . 5}-\mathbf{4 . 5} \mathbf{H z} \\
\text { decreases in } \\
\text { third part }\end{array}$} & \multicolumn{3}{|c|}{$\begin{array}{l}\text { ncreases in } \\
\text { third part }\end{array}$} \\
\hline $\mathbf{L H}$ & $\mathbf{M}$ & RH & $\mathbf{L H}$ & $\mathbf{M}$ & RH \\
\hline 6 & - & - & - & - & - \\
\hline 24 & - & 6 & - & - & - \\
\hline 17 & - & 5 & 1 & - & - \\
\hline 11 & - & 7 & 1 & - & - \\
\hline- & - & 6 & - & - & - \\
\hline 2 & - & 1 & 7 & - & - \\
\hline- & - & 1 & - & - & - \\
\hline- & - & - & 5 & - & - \\
\hline- & - & - & 18 & - & - \\
\hline- & - & - & 13 & - & - \\
\hline 25 & - & 18 & - & - & - \\
\hline
\end{tabular}

LH: left hemisphere, RH: right hemisphere, M: midline voxels. Numbers in italics indicate activity increases in the respective frequency / frequency range and condition, normal font numbers indicate activity decreases.

\subsection{Final rest compared to meditation and initial rest}

Final rest showed decreases compared to meditation in large areas of two frequency ranges (1.5-7 Hz and 13.5-14.5 Hz), and also decreases compared to initial rest in the frequency ranges of 1.0-7 Hz and 13.5-14.5 Hz (Table 1, right). 


\section{Discussion}

The main goal of the present study was to look at changes in EEG sources (i.e. activity in specific brain areas) during BKRYM meditation compared to rest. Arguably, initial rest before meditation is closer to everyday resting than final rest immediately following a meditation session. Therefore, our main focus was on the comparison of meditation with initial rest. In a more exploratory analysis, final rest was included to evaluate possible lingering effects of the meditation session. Further, the meditation session data was partitioned into thirds and pairwise compared as a means to find possible indications of arousal differences due to the passing of time. First, we will discuss the results of the main comparison between meditation and initial rest.

\subsection{Reduced inhibition}

During BKRYM ‘seed stage meditation’, the inhibitory delta band activity (Niedermeyer \& Lopes da Silva, 1993) decreased in a large bilateral frontal and central cluster that extended into left-hemispheric temporal and parietal areas (Figure 1, Table 1 (left) and Table 2). Thus, we see reduced functional inhibition, which likely implies increased activation (processing) in these affected areas during meditation as compared to task-free rest. These areas sub-serve functions such as empathy (bilateral (pre-)frontal: Seitz, Nickel, \& Azari, 2006), behavioral inhibition / executive control (dorsolateral prefrontal cortex - DLPFC: Kübler, Dixon, \& Garavan, 2006; Van Oort et al., 2017), visuo-spatial cognition and spatial information processing (middle frontal, BAs 9/46: Leung, Gore, \& Goldman-Rakic, 2002), somatosensory processing (homunculus) (BAs 3,1,2) and semantic language processing (BAs 20,21,22,44,45,6: Bookheimer, 2002). As hypothesized, at the level of affected brain networks, we see an activation of the central executive network (CEN) (Van Oort et al., 2017) with decreased delta activity in the DLPFC (BAs 9,10 46), the frontal eye fields (FEF; BA 8) and the posterior parietal cortex (PPC; BA 7). Sustaining attention during meditation has been reported to keep the DLPFC as part of the CEN activated (Hasenkamp, Wilson-Mendenhall, Duncan, \& Barsalou, 2012).

Our results fit well the subjective experience involved in seed stage meditation. The increased behavioral inhibition is expected as the meditators sit still and relaxed during meditation. Increased semantic language processing could be the result of the auto-suggestive nature of the meditation process, since the meditators internally verbalize the steps involved in achieving each stage of the meditation. The left-sided activation of language areas (BAs 44/45 and 21/22) possibly reflects the logical reasoning involved in following the sequence of auto-suggestive sentences (Bookheimer, 2002; Caplan \& Dapretto, 2001).

We found increased activation in the somatosensory cortex (homunculus, BAs 3,2,1). On one hand, this is a bit surprising, as meditators do not actively pay attention to their body. One explanation for this finding could be that during resting, the practitioners actively were inhibiting (higher delta activity) the somatosensory cortex, as they were instructed to sit quietly and not move. During meditation, there was no longer any special focus on relaxation or on not moving and the activity in 
somatosensory cortex went back to more normal levels, i.e. resulting in reduced delta activity as compared to resting. On the other hand, the $20 \%$ of voxels showing the highest t-values for reduced delta activity were located in a small cluster with average MNI coordinates $(44,1,51 \mathrm{~mm})$ in the right hemisphere and $(-60,-28,32 \mathrm{~mm})$ in the left hemisphere, corresponding to the face areas on the homunculus (Roux, Djidjeli, \& Durand, 2018). This might be the result of focusing the attention between the eyes as an important stage during the meditation. The increased visuo-spatial cognition and spatial information storage might result from having the eyes opened during the meditation. This allows the meditator to keep an image of himself/herself in relation to the tapestry on the wall that he/she gazes at. It is even conceivable that the nature of the meditation itself fosters spatial processing. Indeed, the meditator attempts to become aware of himself/herself as a soul, a point of light between the eyes that becomes increasingly distanced to the material world. Also, the Supreme Soul is envisioned as separate from the soul before letting the perfect qualities of the Supreme Soul permeate the soul, which implies a direction. This latter part of sensing the perfect qualities of the Supreme Soul and attempting to let them permeate the self, might explain the apparently enhanced emphatic processing during meditation.

\subsection{Increased facilitation}

Power in the low alpha frequencies $(7.0$ to $9.5 \mathrm{~Hz})$ increased during meditation in a cluster of voxels with the cluster being largest at $9.0 \mathrm{~Hz}$ (Figure 1). While upper alpha frequencies have been considered inhibitory (Bazanova \& Vernon, 2014; Klimesch, Doppelmayr, Russegger, Pachinger, \& Schwaiger, 1998; Klimesch et al., 1999), we consider lower alpha frequencies as facilitatory and therefore as activation. This cluster encompassed the bilateral posterior (and to a lesser degree the right anterior) cingulate, extended bilaterally to the parahippocampal gyrus and the superior temporal gyrus, the bilateral insula, the fusiform gyrus, the inferior parietal lobule, bilateral lingual gyrus, and occipitally and bilaterally to the cuneus and precuneus. How does an activation of these brain areas relate to soul-conscious meditation?

The left BA 37 and BA 19 bilaterally have been implicated in mental imagery (D'Esposito et al., 1997), as were BAs 40 and 7 (Knauff, Kassubek, Mulack, \& Greenlee, 2000) and 18 (De Volder et al., 2001). Soul-conscious meditation has a strong focus on mental imagery as the practitioner imagines himself/herself as a point of light between the eyes, as distant from his/her body and the world and witnessing the light of the Supreme Soul.

The involvement of the insula (BA 13) might result from imagining the feeling of peacefulness as the insula processes states of feeling cortically (Damasio, Damasio, \& Tranel, 2012). The perirhinal cortex (BA 36) processes semantic memory (Davies, Graham, Xuereb, Williams, \& Hodges, 2004) as do other parts of the temporal lobe (BAs 20, 21 and 22) (Bookheimer, 2002) and their activation could reflect the meditators' focus on the concept of peacefulness and related memories. One of these areas was activated by decreased delta (BA 21), one by increased low alpha (BA 36) and some by both frequency ranges (BAs 13, 20, 22). Considering both frequency ranges as activations, what activation patterns appear across frequency ranges? 


\subsection{Combined activations}

Across delta and low alpha, several networks were activated. The mirroring or experience sharing network (for a metaanalysis see Molenberghs, Cunnington, \& Mattingley, 2012) was activated in its classical regions (ventral premotor cortex, inferior frontal gyrus, inferior parietal lobule) and also in regions associated especially with mirroring emotional expression (insula and cingulate cortex) (Molenberghs et al., 2012). An important part of BKRYM seed stage meditation is for the practitioner trying to mirror the feeling of peacefulness perceived in the Supreme Soul and let it permeate his/her own soul. This could explain the activation of this network.

Thirty-five $\%$ of all significant voxels across delta and low alpha belonged to the task-positive network (BAs 6, 19, 37, 40, 46) and twenty-two \% to the task-negative network (BAs 8, 10, 20, 21, 30, 31, 39) (Fox et al., 2005). While the activation of the task-positive network confirms our hypothesis, the activation of the task-negative network was unexpected. These two networks are typically anti-correlated (Fox et al., 2005; Fukunaga et al., 2006), except for states of non-dual awareness during deep meditation as proposed by Josipovic (2014). The task-negative network (or default mode network - DMN, Raichle et al., 2001) has been related to mind wandering (Mason et al., 2007), episodic memory processing (Buckner, Andrews-Hanna, \& Schacter, 2008; Greicius, Srivastava, Reiss, \& Menon, 2004) and conceptual processing (Binder et al., 1999). Interestingly, these processes are all important for maintaining the sense of self (Gusnard, Akbudak, Shulman, \& Raichle, 2001; Lou et al., 2004). Many meditation practices tend to weaken the sense of self and are accompanied by a deactivation of the DMN (e.g. Brewer et al., 2011; Garrison, Zeffiro, Scheinost, Constable, \& Brewer, 2015). Soul consciousness though has a strong focus on the self, as it relates the self to the everyday world, the own body, the point between the eyes and the Supreme Soul during its different stages. Self-related processing is known to activate the DMN (Buckner et al., 2008; Raichle et al., 2001).

The areas belonging to the task-positive network have been associated with different aspects of attention (Corbetta, Patel, \& Shulman, 2008; Posner \& Petersen, 1990). Shifting the attention back to the focus of the meditation after noticing mind wandering as well as sustaining the attention on the focus of meditation has been associated with activation in the taskpositive network (Hasenkamp et al., 2012).

It is interesting that the two networks are both activated during BKRYM seed stage meditation. There is no subjective evidence for a state of non-dual awareness during the different stages of the meditation, except maybe for the very last stage. So, they should rather be anti-correlated. It is conceivable that these simultaneous activations are due to the averaging over the different stages of the meditation. Possibly, at different times during the meditation, there is an anti-correlation of the networks. This needs to be disentangled in future studies by differentiating between the different stages of meditation. Across all states, BKRYM seed stage meditation seems to involve the task-negative network with its strong focus on selfreferential processing and the task-positive network with its need to shift attention to and sustain it on the task of going through the different stages of the meditation. 
unclear why certain brain areas were activated by reduced delta activity and others by increased low alpha activity. A few regions showed activations in both frequency ranges (Table 1). Possibly, studying separately the different states of BKRYM seed stage meditation could shed some light on this issue.

How could the present findings relate to the reported benefits of meditation in general and BKRYM in particular? It has been proposed that associative learning processes through imagination help promote change and well-being (Reddan, Wager, \& Schiller, 2018). It is conceivable that the mechanism leading to change and enhanced well-being through the continued practice of soul-conscious meditation lies in imagining oneself as a soul, a light point between the eyes and linking the own soul (oneself) with the Supreme Soul, mirroring its pure attributes (such as peacefulness) and the reported underlying brain-electric activations.

\subsection{Probing for time effects}

To probe for effects of passing time (e.g. changes in arousal) over the 30-min meditation session, the meditation data was partitioned into three blocks, each covering 10 minutes of the meditation. The middle part did not differ from the first or third part. There were slight changes from the first to the third part of the meditation though. These changes were decreases in delta $(1.5-4.5 \mathrm{~Hz})$ activity in somatosensory, premotor and motor cortices (BAs 1-6) as well as the inferior parietal lobule (BA 40) and increases at $26 \mathrm{~Hz}$ in the cingulate cortex (BAs 23, 24, 31), and the premotor and supplementary motor area (BA 6). All these changes also show up when comparing the complete meditation session to initial rest. These changes thus slightly increase with progression of the 30-min meditation session.

\subsection{Comparisons with final rest}

Meditation shows different changes compared to final rest than it does to initial rest. Most noteworthy is the decrease of upper alpha (11.0-14.5 Hz) frequencies in final rest, but also the decrease of activity in the delta and theta $(1.5-7.0 \mathrm{~Hz})$ frequencies in many areas. Thus, it is apparent that coming out of meditation is different from going into meditation. This has already been described in a study on functional connectivity in meditators from 5 different meditation traditions (Lehmann et al., 2012). On a side note, the differential involvement of low alpha during meditation compared to initial rest and upper alpha during final rest compared to meditation seems to imply that low and upper alpha reflect different processes and caution should be applied when analyzing a single broad alpha band.

At this point, we do not want to over-interpret these findings, because final rest immediately followed meditation without any break, except for the experimenter prompting the participant to stop meditating. It seems reasonable that large parts of the brain show changes during this transition back to a normal state. To study the change from meditation to rest after meditation, future studies should record rest during a longer time period after meditation or during several intervals within a couple of hours after meditation. This would help elucidate how the brain returns to its normal, everyday state of mind after an extended meditation session. 
same holds for the comparisons of final to initial rest. Final rest differs largely from initial rest in almost all brain areas, thus possibly showing a lingering effect of the meditation session combined with a reorganization of brain activity to normal processing. Not knowing when the return to a normal state is complete, looking only at these first 3 minutes seems insufficient to draw any useful conclusions.

\subsection{Limitations}

We note some limitations of this study. First, our practitioners were all adept Brahma Kumaris meditators. As such, they practice a certain lifestyle that could very well have an influence on their general state of mind, irrespective of the seed stage meditation studied here. This lifestyle includes celibacy, a vegetarian diet and a strict daily routine (e.g. getting up early for morning meditation).

Second, Mount Abu is situated at 1,722 $\mathrm{m}$ above sea and the altitude could have influenced the EEG patterns. Also, the recordings were performed during a winter retreat. We note that all participants had 3 to 5 days of acclimatization before the recording. The more intense meditation schedule during the retreat might also have influenced the results. Although, these effects should all be mitigated by our intra-subject comparisons.

Third, the recording protocol did not control for the different stages of meditation. Each meditator used his/her idiosyncratic pacing. To disentangle the brain electric characteristics of the different stages, these should be triggered by the experimenter in a controlled fashion in future studies.

Fourth, to study how the brain activity returns to normal after an extended meditation session, future studies should record rest after meditation over a prolonged period.

\subsection{Conclusion}

In sum, the BKRYM seed stage meditation with open eyes showed activations in brain areas sub-serving the subjective experience of the practitioners during the different stages of meditation. The modulated areas were part of the CEN, the DMN and the task-positive network. They inhibit movement, foster and modulate attention to stay on the task of moving through the different stages of the meditation and enable self-related processing for experiencing the soul as a point of light between the eyes, distant from the everyday world, and endowing it with the properties (i.e. peacefulness) of the Supreme Soul. 


\section{Ethical approval}

All the procedures performed in this study were in accordance with the ethical standards of the Institutional ethics committee of Rajayoga Education and Research Foundation (RERF) and with the 1964 Helsinki declaration and its later amendments or comparable ethical standards.

\section{Funding}

This research did not receive any specific grant from funding agencies in the public, commercial, or

not-for-profit sectors.

\section{Disclosure}

The authors declare that they have no conflict of interest.

\section{References}

Bazanova, O., \& Vernon, D. (2014). Interpreting EEG alpha activity. Neuroscience \& Biobehavioral Reviews, 44, 94-110. Binder, J. R., Frost, J. A., Hammeke, T. A., Bellgowan, P., Rao, S. M., \& Cox, R. W. (1999). Conceptual processing during the conscious resting state: a functional MRI study. Journal of cognitive neuroscience, 11(1), 80-93. Birch, J. (2013). Rājayoga: The Reincarnations of the King of All Yogas. International Journal of Hindu Studies, 17(3), 399-442.

Bookheimer, S. (2002). Functional MRI of language: new approaches to understanding the cortical organization of semantic processing. Annual review of neuroscience, 25(1), 151-188.

Brahmakumaris. (1986). Positive Health - Rajayoga meditation for stress-free peaceful and healthy life. Delhi, India: Prajapitha Brahma Kumaris Ishwarya Vishwa Vidyalaya.

Brewer, J. A., Worhunsky, P. D., Gray, J. R., Tang, Y.-Y., Weber, J., \& Kober, H. (2011). Meditation experience is associated with differences in default mode network activity and connectivity. Proceedings of the National Academy of Sciences, 108(50), 20254-20259.

Buckner, R. L., Andrews-Hanna, J. R., \& Schacter, D. L. (2008). The brain's default network: anatomy, function, and relevance to disease. Annals of the New York Academy of Sciences, 1124(1), 1-38.

Caplan, R., \& Dapretto, M. (2001). Making sense during conversation: an fMRI study. Neuroreport, 12(16), $3625-3632$. Corbetta, M., Patel, G., \& Shulman, G. L. (2008). The reorienting system of the human brain: from environment to theory of mind. Neuron, 58(3), 306-324.

D'Esposito, M., Detre, J. A., Aguirre, G. K., Stallcup, M., Alsop, D. C., Tippet, L. J., et al. (1997). A functional MRI study of mental image generation. Neuropsychologia, 35(5), 725-730.

Dahl, C. J., Lutz, A., \& Davidson, R. J. (2015). Reconstructing and deconstructing the self: cognitive mechanisms in meditation practice. Trends in cognitive sciences, 19(9), 515-523.

Damasio, A., Damasio, H., \& Tranel, D. (2012). Persistence of feelings and sentience after bilateral damage of the insula. Cerebral Cortex, 23(4), 833-846.

Davies, R., Graham, K. S., Xuereb, J. H., Williams, G. B., \& Hodges, J. R. (2004). The human perirhinal cortex and semantic memory. European Journal of Neuroscience, 20(9), 2441-2446. 
Sharma, Achermann, Panwar, Sahoo, Angarai Ganesan, Pascual-Marqui, Faber: Brain-electric activity during eyes open

Volder, A. G., Toyama, H., Kimura, Y., Kiyosawa, M., Nakano, H., Vanlierde, A., et al. (2001). Auditory triggered mental imagery of shape involves visual association areas in early blind humans. Neuroimage, 14(1), 129-139.

Evans, A., \& Collins, D. (1993). A 305-member MRI-based stereotaxic atlas for CBF activation studies. Paper presented at the Journal of Nuclear Medicine.

Fox, M. D., Snyder, A. Z., Vincent, J. L., Corbetta, M., Van Essen, D. C., \& Raichle, M. E. (2005). The human brain is intrinsically organized into dynamic, anticorrelated functional networks. Proceedings of the National Academy of Sciences, 102(27), 9673-9678.

Frei, E., Gamma, A., Pascual-Marqui, R., Lehmann, D., Hell, D., \& Vollenweider, F. X. (2001). Localization of MDMAinduced brain activity in healthy volunteers using low resolution brain electromagnetic tomography (LORETA). Human brain mapping, 14(3), 152-165.

Fukunaga, M., Horovitz, S. G., van Gelderen, P., de Zwart, J. A., Jansma, J. M., Ikonomidou, V. N., et al. (2006). Largeamplitude, spatially correlated fluctuations in BOLD fMRI signals during extended rest and early sleep stages. Magnetic resonance imaging, 24(8), 979-992.

Garrison, K. A., Zeffiro, T. A., Scheinost, D., Constable, R. T., \& Brewer, J. A. (2015). Meditation leads to reduced default mode network activity beyond an active task. Cognitive, Affective, \& Behavioral Neuroscience, 15(3), $712-720$.

Greicius, M. D., Srivastava, G., Reiss, A. L., \& Menon, V. (2004). Default-mode network activity distinguishes Alzheimer's disease from healthy aging: evidence from functional MRI. Proceedings of the National Academy of Sciences, 101(13), 4637-4642.

Gusnard, D. A., Akbudak, E., Shulman, G. L., \& Raichle, M. E. (2001). Medial prefrontal cortex and self-referential mental activity: relation to a default mode of brain function. Proceedings of the National Academy of Sciences, 98(7), 4259-4264. Hasenkamp, W., Wilson-Mendenhall, C. D., Duncan, E., \& Barsalou, L. W. (2012). Mind wandering and attention during focused meditation: a fine-grained temporal analysis of fluctuating cognitive states. Neuroimage, 59(1), 750-760.

Josipovic, Z. (2010). Duality and nonduality in meditation research. Consciousness and cognition, 19(4), 1119-1121. Josipovic, Z. (2014). Neural correlates of nondual awareness in meditation. Annals of the New York Academy of Sciences, 1307(1), 9-18.

Keng, S.-L., Smoski, M. J., \& Robins, C. J. (2011). Effects of mindfulness on psychological health: A review of empirical studies. Clinical psychology review, 31(6), 1041-1056.

Kiran, Chalana, H., Arora, A. K., \& Girgila, K. K. (2014). Effect of short term Rajyoga meditation on anxiety and depression. Pakistan Journal of Physiology, 10(1-2), 18-20.

Klimesch, W., Doppelmayr, M., Russegger, H., Pachinger, T., \& Schwaiger, J. (1998). Induced alpha band power changes in the human EEG and attention. Neuroscience letters, 244(2), 73-76.

Klimesch, W., Doppelmayr, M., Schwaiger, J., Auinger, P., \& Winkler, T. (1999). Paradoxical'alpha synchronization in a memory task. Cognitive Brain Research, 7(4), 493-501.

Knauff, M., Kassubek, J., Mulack, T., \& Greenlee, M. W. (2000). Cortical activation evoked by visual mental imagery as measured by fMRI. Neuroreport, 11(18), 3957-3962.

Kübler, A., Dixon, V., \& Garavan, H. (2006). Automaticity and reestablishment of executive control—An fMRI study. Journal of cognitive neuroscience, 18(8), 1331-1342.

Lehmann, D., Faber, P. L., Tei, S., Pascual-Marqui, R. D., Milz, P., \& Kochi, K. (2012). Reduced functional connectivity between cortical sources in five meditation traditions detected with lagged coherence using EEG tomography. Neuroimage, 60(2), 1574-1586.

Leung, H.-C., Gore, J. C., \& Goldman-Rakic, P. S. (2002). Sustained mnemonic response in the human middle frontal gyrus during on-line storage of spatial memoranda. Journal of cognitive neuroscience, 14(4), 659-671.

Lou, H. C., Luber, B., Crupain, M., Keenan, J. P., Nowak, M., Kjaer, T. W., et al. (2004). Parietal cortex and representation of the mental self. Proceedings of the National Academy of Sciences, 101(17), 6827-6832.

Lutz, A., Slagter, H. A., Dunne, J. D., \& Davidson, R. J. (2008). Attention regulation and monitoring in meditation. Trends in cognitive sciences, 12(4), 163-169.

Mason, M. F., Norton, M. I., Van Horn, J. D., Wegner, D. M., Grafton, S. T., \& Macrae, C. N. (2007). Wandering minds: the default network and stimulus-independent thought. Science, 315(5810), 393-395.

Meshram, K., \& Meshram, A. (2016). Usefulness of Rajyoga meditation in assessment of general well being of badminton players an experimental study. Int J Pharm Biol Sci, 7(1), 161-166.

Misra, N., Gupta, A., Alreja, S., \& Prakash, O. (2013). Effect of Raj Yoga meditation on affective \& cognitive functions. International Journal of Heath Sciences and Research, 3(2), 38-46.

Molenberghs, P., Cunnington, R., \& Mattingley, J. B. (2012). Brain regions with mirror properties: a meta-analysis of 125 human fMRI studies. Neuroscience \& Biobehavioral Reviews, 36(1), 341-349. 
Sharma, Achermann, Panwar, Sahoo, Angarai Ganesan, Pascual-Marqui, Faber: Brain-electric activity during eyes open

Naik, A., Patel, S., Biswas, D., \& Verma, M. (2016). Effect of Rajyoga meditation on intelligence quotient of attention deficit hyperactivity disorder. J Yoga Phys Ther, 6(242), 2.

Nair, A. K., Sasidharan, A., John, J. P., Mehrotra, S., \& Kutty, B. M. (2017). Just a minute meditation: Rapid voluntary conscious state shifts in long term meditators. Consciousness and cognition, 53, 176-184.

Nash, J. D., \& Newberg, A. (2013). Toward a unifying taxonomy and definition for meditation. Frontiers in psychology, 4, 806.

Nichols, T. E., \& Holmes, A. P. (2002). Nonparametric permutation tests for functional neuroimaging: a primer with examples. Human brain mapping, 15(1), 1-25.

Niedermeyer, E., \& Lopes da Silva, F. (1993). Electroencephalography: basic principles, clinical applications and related fields. (3rd ed.). Baltimore, MD: Williams and Wilkins,.

Nuwer, M. R., Comi, G., Emerson, R., Fuglsang-Frederiksen, A., Guérit, J.-M., Hinrichs, H., et al. (1998). IFCN standards for digital recording of clinical EEG. Electroencephalography and clinical Neurophysiology, 106(3), $259-261$.

Panda, R., Bharath, R. D., Upadhyay, N., Mangalore, S., Chennu, S., \& Rao, S. L. (2016). Temporal dynamics of the default mode network characterize meditation-induced alterations in consciousness. Frontiers in human neuroscience, $10,372$.

Pascual-Marqui, R. D. (2007). Discrete, 3D distributed, linear imaging methods of electric neuronal activity. Part 1: exact, zero error localization. arXiv preprint arXiv:0710.3341.

Pascual-Marqui, R. D., Lehmann, D., Koenig, T., Kochi, K., Merlo, M. C., Hell, D., et al. (1999). Low resolution brain electromagnetic tomography (LORETA) functional imaging in acute, neuroleptic-naive, first-episode, productive schizophrenia. Psychiatry Research: Neuroimaging, 90(3), 169-179.

Pascual-Marqui, R. D., Michel, C. M., \& Lehmann, D. (1994). Low resolution electromagnetic tomography: a new method for localizing electrical activity in the brain. International Journal of psychophysiology, 18(1), 49-65.

Posner, M. I., \& Petersen, S. E. (1990). The attention system of the human brain. Annual review of neuroscience, 13(1), 2542.

Raffone, A., \& Srinivasan, N. (2010). The exploration of meditation in the neuroscience of attention and consciousness. Cogn Process, 11, 1-7.

Raichle, M. E., MacLeod, A. M., Snyder, A. Z., Powers, W. J., Gusnard, D. A., \& Shulman, G. L. (2001). A default mode of brain function. Proceedings of the National Academy of Sciences, 98(2), 676-682.

Rajoria, K., \& Singh, S. K. (2017). Therapeutic benefits of Raj Yoga-A review. Indian Journal of Traditional Knowledge, 16(Suppl), 88-95.

Ramesh, M., Sathian, B., Sinu, E., \& Kiranmai, S. R. (2013). Efficacy of rajayoga meditation on positive thinking: An index for self-satisfaction and happiness in life. Journal of clinical and diagnostic research: JCDR, 7(10), 2265.

Ramsay, T., \& Manderson, L. (2011). Resilience, spirituality and posttraumatic growth: Reshaping the effects of climate change. In Climate change and human well-being (pp. 165-184): Springer.

Ramsay, T., Manderson, L., \& Smith, W. (2010). Changing a mountain into a mustard seed: Spiritual practices and responses to disaster among New York Brahma Kumaris. Journal of Contemporary Religion, 25(1), 89-105.

Reddan, M. C., Wager, T. D., \& Schiller, D. (2018). Attenuating neural threat expression with imagination. Neuron, 100(4), 994-1005. e1004.

Roux, F. E., Djidjeli, I., \& Durand, J. B. (2018). Functional architecture of the somatosensory homunculus detected by electrostimulation. The Journal of physiology, 596(5), 941-956.

Seitz, R. J., Nickel, J., \& Azari, N. P. (2006). Functional modularity of the medial prefrontal cortex: involvement in human empathy. Neuropsychology, 20(6), 743.

Sharma, K., Chandra, S., \& Dubey, A. K. (2018). Exploration of lower frequency EEG dynamics and cortical alpha asymmetry in long-term rajyoga meditators. International journal of yoga, 11(1), 30.

Sukhsohale, N. D., \& Phatak, M. S. (2012). Effect of short-term and long-term Brahmakumaris Raja Yoga meditation on physiological variables. Indian J Physiol Pharmacol, 56(4), 388-392.

Telles, S., \& Desiraju, T. (1993). Autonomic changes in Brahmakumaris Raja yoga meditation. International Journal of Psychophysiology, 15(2), 147-152.

Travis, F., \& Shear, J. (2010). Focused attention, open monitoring and automatic self-transcending: categories to organize meditations from Vedic, Buddhist and Chinese traditions. Consciousness and cognition, 19(4), 1110-1118.

Van Oort, J., Tendolkar, I., Hermans, E., Mulders, P., Beckmann, C., Schene, A., et al. (2017). How the brain connects in response to acute stress: A review at the human brain systems level. Neuroscience \& Biobehavioral Reviews, 83, $281-297$. 